\title{
PARÁMETROS SEMINALES EN HOMBRE FÉRTILES DE DOS POBLACIONES SURAMERICANAS
}

\author{
Jesus Berdugo 1,3, Fernando Andrade-Rocha y y Walter Cardona-Maya'. \\ 'Grupo Reproducción. Universidad de Antioquia. Medellín. Colombia. \\ 2 Unidad de Investigación Seminal. Laboratorio Homero Soares. Petrópolis. RJ. Brasil.
}

Resumen.- OBJETIVO: Este estudio retrospectivo compara los parámetros seminales de hombres fértiles de dos ciudades suramericanas Medellín, Colombia y Petrópolis, Brasill) con el fin de investigar sus diferencias en algunos parámetros seminales.

MÉTODOS: Se evaluó el volumen seminal usando un tubo graduado, la movilidad progresiva ( $a+b$ ) usando un microscopio de luz (4OX) y la concentración espermática usando la cámara de Neubaver.
RESULTADOS: Se observó que los hombres fértiles de Medellín presentan una disminución significativa del volumen ( $p<0.0001)$, en contraste los individuos de Petrópolis presentaron una disminución significativa de la movilidad progresiva ( $p<0.0001)$. De otro lado, no se encontró diferencia en la concentración espermática (p>0.05).

CONCLUSIONES: Este estudio mostró diferencias en los parámetros espermáticos entre los hombres fértiles de dos poblaciones suramericanas. Nosotros pensamos que estas diferencias podrían ser atribuidas a las variaciones geográficas, como han sido observadas en otros países. Sin embargo, nuevos estudios son necesarios para investigar este fenómeno y sus causas.

Palabras clave: Análisis seminal. Fertilidad poblacional. Calidad seminal. Espermatozoide. Características espermáticas. Volumen.

Summary.- OBJECTIVES: This retrospective study compared semen parameters from fertile men of two South American cities (Medellín-Colombia and Petrópolis-Brazill to investigate their differences in some semen parameters.

METHODS: We evaluated semen volume using a graduated tube, total progressive motility $(a+b)$ using light microscopy (40X) and sperm concentration using a Neubaver Counting Chamber.

RESULTS: We observed that fertile men from Medellin presented a significantly lower volume $(p<0.0001)$, whereas individuals from Petrópolis presented a significantly lower percentage of total progressive motility 
$(p<0.0001)$. On the other hand, no difference was found in sperm concentration ( $p>0.05)$.

CONCLUSIONS: In conclusion, this study showed differences in semen parameters between fertile men of these South American populations. We think that these differences could be attributed to the geographical variations, like those observed in other countries. However, new studies are required to investigate this phenomenon and their causes.

Keywords: Population fertility. Semen quality. Spermatozoa.Sperm characteristics. Volume.

\section{INTRODUCCIÓN}

Estudios demográficos realizados en hombres fértiles de Estados Unidos y Europa, han mostrado que existen diferencias en los parámetros seminales intra y entre las poblaciones (1-4), por lo tanto es posible sugerir que existen variaciones en el potencial fértil de las poblaciones en diferentes partes del mundo y que estas pueden ser observadas por hallazgos como diferencias en los parámetros seminales. Sin embargo, los resultados obtenidos en cada población son muy difíciles de extrapolar a otra, debido a que generalmente los parámetros seminales y la tasa de fertilidad son evaluadas en muestras homogéneas de individuos (individuos que van a ser vasectomizados, donantes de semen 0 individuos sanos con fertilidad probada) los cuales no necesariamente reflejan el estado reproductivo de la población general. Adicionalmente, estos estudios no tienen en cuenta limitaciones relacionadas con la población como abstinencia sexual, factores ambientales, estilo de vida y la metodología usada para el análisis seminal.

De otro lado, es importante recalcar que un hombre con parámetros seminales alterados según la Organización Mundial de la Salud (OMS) puede concebir $(5,6)$, mientras que otros con todos los parámetros normales no lo logran (7), las causas de este fenómeno aún son desconocidas. Los estudios sobre parámetros seminales en las poblaciones y su variación relacionada con la geografía, han sido realizados principalmente en el hemisferio norte (1-4), muy poca información existe sobre este tema en las poblaciones del trópico, donde no hay estaciones. El objetivo del presente estudio fue comparar los parámetros seminales de hombres fértiles de dos ciudades localizadas en el trópico Sur Americano, Medellín, Colombia y Petrópolis, Brasil.

\section{MATERIALES Y MÉTODOS}

\section{Muestras de Semen}

Este estudio retrospectivo evaluó los registros de hombres fértiles antes de realizarse la vasectomía en la Clínica de Fertilidad Masculina de Medellín Colombia entre los años 1999 y 2004 (8) y en el Laboratorio de Investigación Seminal Homero Soares Ramos, Petrópolis-Brazil. Todos las parejas de los individuos colombianos y brasileños incluidos en el estudio habían tenido un parto en el último año, y el período de búsqueda de ese embarazo (relaciones sexuales sin protección) no fue superior a 12 meses. Individuos con alteraciones testiculares (varicocele, criptorquidismo, desordenes hormonales), traumas testiculares (traumas, orquitis y torsiones), leucocitospermia, bacteriospermia, diabetes, hipertensión, consumo de drogas o enfermedades agudas fueron excluidos del estudio. Este estudio fue aprobado por los comités de ética del Centro de Investigaciones Médicas de la Universidad de Antioquia y del Laboratorio Homero Soares.

\section{Procedimiento de Laboratorio}

Se evaluaron los parámetros seminales de 113 hombres fértiles colombianos y 84 brasileños en el laboratorio de análisis seminal del Grupo Reproducción, Universidad de Antioquia y en la Unidad de Evaluación Seminal del Laboratorio Homero Soares Ramos, respectivamente. Las muestras de semen fueron tomadas por masturbación luego de $4.0 \pm 0.1$ días de abstinencia sexual en los colombianos y de $5.3 \pm 0.7$ días en los brasileños. A estas muestras se les determinó, el volumen, la movilidad progresiva (a + b) y la concentración, utilizando un tubo graduado (margen de error $0,1 \mathrm{ml}$ ), observación directa al microscopio de luz $(40 x)$ y la cámara de Neubaver, respectivamente, siguiendo las recomendaciones de la OMS para análisis seminal de 1999 (13). Todas las muestras fueron analizadas antes de 60 minutos de haber sido tomadas, posterior a su licuefacción.

\section{Análisis Estadístico}

A los resultados obtenidos en los grupos se les aplicó un prueba de t-test no pareada, utilizando el programa Graph Pad Prism $\AA$ version 4.0. Diferencias de $p<0.05$ fueron consideradas como significativas.

\section{RESULTADOS}

Los parámetros seminales evaluados en los hombres fértiles colombianos y brasileños se muestran en la Tabla I (promedio \pm DS). Las comparaciones entre las poblaciones estudiadas muestran que el volumen es significativamente menor en los hombres 
colombianos $(p<0.0001)$, mientras que los brasileños tienen una menor movilidad progresiva $(p<0.0001)$. No se encontraron diferencias en la concentración espermática $(p<0.05)$.

\section{DISCUSIÓN}

A nuestro mejor conocimiento, este es el primer estudio que compara los parámetros seminales en dos muestras de hombres fértiles suramericanas. En el presente estudio se encontraron diferencias en el volumen y la movilidad progresiva, pero no en la concentración espermática. Otros autores han informado diferencias en la calidad seminal de hombres fértiles debido a variaciones geográficas en otras partes del mundo como: Europa (1, 2), Japón (4), y Estados Unidos de América (3). Sin embargo, en América del Sur solo existen tres estudios poblacionales: Bahamondes y cols., (9) y Matheus y cols., (10), en Brasil y Tortolero y cols., (1 1) en Venezuela. Desafortunadamente sus resultados son muy difíciles de comparar debido a diferencias en las muestras y en el diseño de los estudios, por ejemplo, el estudio venezolano fue realizado en hombres infértiles, mientras que los brasileños usaron poblaciones fértiles.

Las variaciones observadas en el volumen seminal en el presente estudio, son similares a las reportadas por otros autores $(1,12)$, dichas diferencias puedan deberse a la posición geográfica de las poblaciones estudiadas, debido a que otras variables como la abstinencia sexual y la metodología usada para el análisis seminal fueron controladas, siguiendo las recomendaciones de la OMS (13). Sin embargo, es importante recalcar que variaciones en la abstinencia sexual podría afectar el volumen seminal (14-16). Adicionalmente, otros factores que podrían afectar el volumen seminal tales como actividad anormal de la próstata o las vesículas seminales, factores sicológicos asociados con la masturbación y pérdida de la muestra (17-19) no fueron tenidos en cuenta en este estudio. A pesar de lo anterior, las diferencias observadas en este estudio pueden ser atribuidas a factores geográficos debido a que se evaluaron hombres fértiles sanos sin anormalidades en sus genitales.

Respecto a la movilidad espermática, se observo que esta fue significativamente menor en la muestra brasilera. Es de anotar que el parámetro que mayor subjetividad presenta en la evaluación seminal es la movilidad, con una gran variación inter-observador (20), una vez mas insistimos en una explicación relacionada es la ubicación geográfica de las ciudades, debido a que la metodología usada fue la misma. Es interesante observar que cuando se compara toda la población evaluada en el laboratorio de Petrópolis el valor obtenido esta por debajo del mínimo aceptado en la población según el manual de la OMS para poblaciones normales (13), 50\% de espermatozoides $(a+b)$, lo cual es congruente con informes previos $(21,22)$ y permiten sugerir la necesidad de discutir el valor de referencia normal para la movilidad espermática. Aunque no es el propósito de este artículo discutir este punto, si debería uno

TABLA I. PARÁMETROS SEMINALES EN HOMBRES FÉRTILES DE COLOMBIA Y BRASIL.

\begin{tabular}{|l|c|c|c|}
\hline Parámetro & Colombia & Brasil & Valor $\mathbf{p}$ \\
\hline Edad (años) & $\mathbf{n = 1 1 3}$ & $\mathbf{n = 8 4}$ & \\
\hline Volumen (ml) & $30.9 \pm 4.2$ & $36.3 \pm 6.5$ & \\
\hline Concentración espermática (106/ml) & $106.4 \pm 7.2$ & $141.2 \pm 12.5$ & $>0.05 \mathrm{a}$ \\
\hline Movilidad progresiva (a $+\mathrm{b}) \%$ & $53.9 \pm 1.6$ & $43.3 \pm 1.4$ & $<0.0001 \mathrm{a}$ \\
\hline
\end{tabular}

Los valores se expresan como promedios \pm SEM. a Distribución normal (t-test no pareado). 
esperar que los hombres fértiles de una población tengan todos parámetros seminales por encima de los aceptados como normales por la OMS.

En el presente estudio no se encontraron diferencias estadísticas en la concentración espermática, sin embargo el valor medio de la muestra brasilera fue mayor. Adicionalmente, ambas poblaciones presentan valores superiores a $20 \times 106$ espermatozoi$\mathrm{des} / \mathrm{mL}$, valor de referencia según la OMS. Los valores encontrados en este estudio son similares a los encontrados en otros estudios $(2,25)$ aunque se han reportado variaciones intra e inter individuos (23) en este parametro debido a su distribución no normal (24).

Las diferencias observadas en los parámetros seminales en otros estudios de hombre fértiles, normalmente se han atribuido a aspectos diferentes al geográfico, tales como: diseño del estudio, población analizada (hombres fértiles, donantes de semen, forma de vida rural o urbana), abstinencia sexual, método de análisis seminal (hemocitometro, análisis computarizado -CASA-, criterios estricto de morfología de Kruger), y a otras variables no tan controladas como fumar, edad, abstinencia sexual, historia de enfermedades de transmisión sexual entre otras $(2,3$, $26,27)$. En consecuencia asumir que lo observado en el presente estudio, es debido únicamente al factor geográfico no es adecuado, y son necesarios más estudios controlando un mayor número de variables que las controladas en el actual.

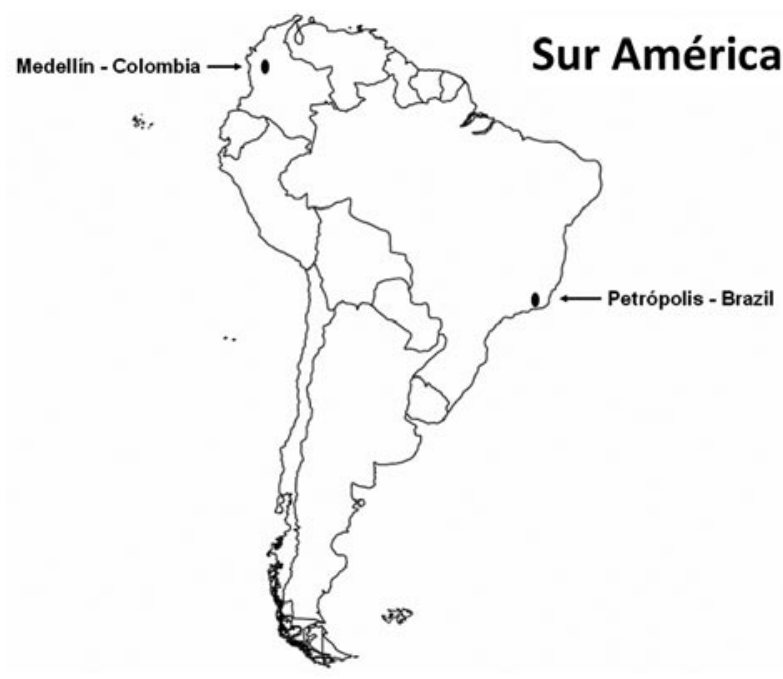

FIGURA 1. Localización geográfica de las ciudades del estudio Medellín y Petrópolis.
La evaluación de rutina para determinar el potencial fértil de un hombre incluye la determinación del volumen, la movilidad y la concentración espermática, y los estudios comparativos existentes en la literatura han informado variaciones geográficas, aún en hombres con fertilidad probada $(1,12)$. Basados en nuestros resultados, se plantea que las diferencias encontradas en volumen y movilidad puedan ser explicadas por factores geográficos. Medellín es una ciudad localizada al noroeste de Colombia, $16^{\circ}$ $\left.15^{\prime} \mathrm{N}\right)$ de latitud y $\left(75^{\circ} 36^{\prime} \mathrm{W}\right)$ de longitud, en el departamento de Antioquia a 1538 metros sobre el nivel del mar con una temperatura que oscila entre 18 y 28 grados celsius.

De otro lado, Petrópolis está localizada en el sureste de Brasil, $\left(22^{\circ} 30^{\prime} \mathrm{S}\right)$ de latitud y $\left(43^{\circ} 10^{\prime} \mathrm{W}\right)$ de longitud, en el estado de Rio de Janeiro, a 830 metros sobre el nivel del mar con una temperatura que oscilia entre 18 y 24 grados Celsius lo que le ha un clima suave tropical subhúmedo, sin épocas secas. Medellín y Petrópolis no presentan estaciones ni tampoco diferencias en sus características socio demográficas, aunque si en el número de habitantes (Medellín 2.223.078 and Petrópolis 302.477) y localización geográfica (Figura 1). Aunque la naturaleza del estudio no permite explicar las diferencias observadas no hay nada que sugiera que estas no puedan ser debidas a factores eminentemente geográficos.

En conclusión, los individuos colombianos y brasileños analizados en este estudio presentan diferencias en el volumen seminal, y la movilidad progresiva, pero no en la concentración. Aunque se utilizaron las sugerencias de la OMS para el análisis seminal, se deben establecer criterios estrictos para eliminar los sesgos en estos estudios. Los resultados presentados en este trabajo, sugieren que la calidad seminal puede variar entre regiones, países e inclusive ciudades, probablemente debido a factores ambientales, étnicos, de estilo de vida, o condiciones geográficas. Sin embargo, es muy importante realizar estudios prospectivos que nos permitan ahondar en las explicaciones a los hechos encontrados.

\section{AGRADECIMIENTOS}

A la Universidad de Antioquia por el apoyo económico. Walter Cardona-Maya es financiado por una beca de COLCIENCIAS. A la Clinica Masculina Profamilia, Medellin, Colombia. 


\section{BIBLIOGRAFÍA Y LECTURAS RECOMENDADAS (*lectura de interés $y$ ** lectura fundamental)}

1. Auger J, Jouannet P. Evidence for regional differences of semen quality among fertile French men. Hum Reprod 1997,12:740-745.

2. Jorgensen N, Andersen AG, Eustache F, et al. Regional differences in semen quality in Europe. Hum Reprod 2001,16:1012-1019.

*3. Swan SH, Brazil C, Drobnis EZ, et al. Geographic differences in semen quality of fertile U.S. males. Environ Health Perspect 2003,111:414-420.

4. Iwamoto T, Nozawa S, Yoshiike M, et al. Semen quality of 324 fertile Japanese men. Hum Reprod 2006,21:760-765.

5. Matorras R, Diez J, Corcostegui B, et al. Spontaneous pregnancy in couples waiting for artificial insemination donor because of severe male infertility. Eur J Obstet Gynecol Reprod Biol 1996,70:175-178.

6. Almagor M, Dan-Goor M, Hovav Y, Yaffe H. Spontaneous pregnancies in severe oligoasthenozoospermia. Hum Reprod 2001,16:1780-1781.

7. Mackenna A. Contribution of the male factor to unexplained infertility: a review. Int J Androl 1995,18 Suppl 1:58-61.

**8. de los Rios J, Cardona WD, Berdugo JA, et al. Los valores espermáticos de 113 individuos con fertilidad reciente no mostraron correlación con los parámetros establecidos por la OMS. Arch Esp Urol 2004,57:147-152.

9. Bahamondes L, Abdelmassih R, J.N. D. Survey of 185 sperm analysis of fertile men in an infertility service. Int J Androl 1979,2:526-533.

10. Matheus M, Sala MA, Barrionovo N, Franceschini SA. Determination of the parameters of human semen in subjects with confirmed fertility. J Bras Ginecol 1986,96:225-229.

*11. Tortolero I, Bellabarba Arata G, Lozano R, Bellabarba C, Cruz I, Osuna JA. Semen analysis in men from Merida, Venezuela, over a 15-year period. Arch Androl 1999,42:29-34.

12. Brazil C, Swan SH, Drobnis EZ, et al. Standardized methods for semen evaluation in a multicenter research study. J Androl 2004,25:635-644.

13. WHO. WHO Laboratory Manual for the Examination of Human Semen and Sperm-Cervical Mucus Interaction. Cambridge: Cambridge University Press; 1999.
14. Padova G, Tita P, Briguglia G, Giuffrida D. Influence of abstinence length on ejaculate characteristics. Acta Eur Fertil 1988,19:29-31.

15. Pellestor F, Girardet A, Andreo B. Effect of long abstinence periods on human sperm quality. Int $\mathbf{J}$ Fertil Menopausal Stud 1994,39:278-282.

16. De Jonge $\mathrm{C}, \mathrm{LaFromboise} \mathrm{M}$, Bosmans $\mathrm{E}, \mathrm{Om}-$ belet $\mathrm{W}, \mathrm{Cox} \mathrm{A}, \mathrm{Nijs} \mathrm{M}$. Influence of the abstinence period on human sperm quality. Fertil Steril 2004,82:57-65.

17. Cooke S, Tyler JP, Driscoll GL. Hyperspermia: the forgotten condition? Hum Reprod 1995,10:367368.

18. Jung A, Schuppe HC, Schill WB. Comparison of semen quality in older and younger men attending an andrology clinic. Andrologia 2002,34:116122.

19. Eskenazi B, Wyrobek AJ, Sloter E, et al. The association of age and semen quality in healthy men. Hum Reprod 2003,18:447-454.

20. Jorgensen N, Auger J, Giwercman A, et al. Semen analysis performed by different laboratory teams: an intervariation study. Int J Androl 1997,20:201208.

*21. Ombelet W, Bosmans E, Janssen M, et al. Semen parameters in a fertile versus subfertile population: a need for change in the interpretation of semen testing. Hum Reprod 1997,12:987-993.

22. Gunalp S, Onculoglu C, Gurgan T, Kruger TF, Lombard CJ. A study of semen parameters with emphasis on sperm morphology in a fertile population: an attempt to develop clinical thresholds. Hum Reprod 2001,16:110-114.

23. Alvarez C, Castilla JA, Martinez L, Ramirez JP, Vergara F, Gaforio JJ. Biological variation of seminal parameters in healthy subjects. Hum Reprod 2003,18:2082-2088.

24. Handelsman DJ. Optimal power transformations for analysis of sperm concentration and other semen variables. J Androl 2002,23:629-634.

25. Fisch H, Goluboff ET, Olson JH, Feldshuh J, Broder SJ, Barad DH. Semen analyses in 1,283 men from the United States over a 25-year period: no decline in quality. Fertil Steril 1996,65:10091014.

26. Contraceptive efficacy of testosterone-induced azoospermia and oligozoospermia in normal men. Fertil Steril 1996,65:821-829.

27. Fisch H, Ikeguchi EF, Goluboff ET. Worldwide variations in sperm counts. Urology 1996,48:909911. 